\title{
Auszeichnung einer hervorragenden Diplomarbeit der Technischen Fachhochschule Wildau mit dem GfPM-Preis 2002
}

\author{
Alfred Hentschel, Stephan Jahnke
}

Jährlich wird durch die Gesellschaft für Produktionsmanagement e. V. (GfPM) für sehr gute Diplomarbeiten auf dem gleichnamigen Fachgebiet der GfPM-Preis an Universitäts- und Fachhochschulabsolventen vergeben. Der Begriff „Produktionsmanagement“ wird durch die GfPM nicht nur auf das Thema „Fertigung“ reduziert, sondern umfasst darüber hinaus auch die Arbeitsvorbereitung, die Fertigungssteuerung, die Logistik, den Einkauf, die Qualitätssicherung, Personal- und Organisationsfragen und die Produktionsinformatik.

Nicht zuletzt gehört mehr als nur oberflächliches Wissen zum Finanz- und Rechnungswesen sowie zum Controlling und zum Produktentstehungsprozess bis hin zu den Vertriebsbelangen dazu.

Die Gesellschaft für Produktionsmanagement wurde 1976 als eingetragener Berufsverband gegründet. Im Brennpunkt stehen gesamtheitliche Aufgaben des Produktions- und produktionsnahen Dienstleistungsmanagements.

Für den GfPM-Preis 2002 wurden für diesen sehr komplexen Anspruch von namhaften Universitäten und Fachhochschulen sehr gute Diplomarbeiten mit einem hohen Bezug zur betrieblichen Praxis eingereicht, von denen in diesem Jahr ein 2. und 3. Preis vergeben wurden. Den 3. Preis erhielt die vorliegende Diplomarbeit mit dem Thema: „Schaffung von Planungsgrundlagen für die Produktionsvorbereitung in der Firma HUCH GmbH Behälterbau Neuruppin unter Beachtung von Kostengesichtspunkten und wirtschaftlichen Losgrößen.“

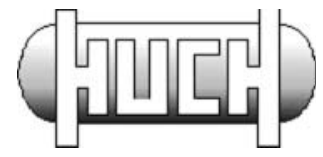

\section{Abb. 1: Firmenlogo}

Dies freut uns um so mehr, weil die Bewerbung dieser Arbeit fuir ein klein- bzw. mittelständisches Unternehmen gegenuiber namhaften Unternehmen und Universitäten den Zuschlag bekommen hat.

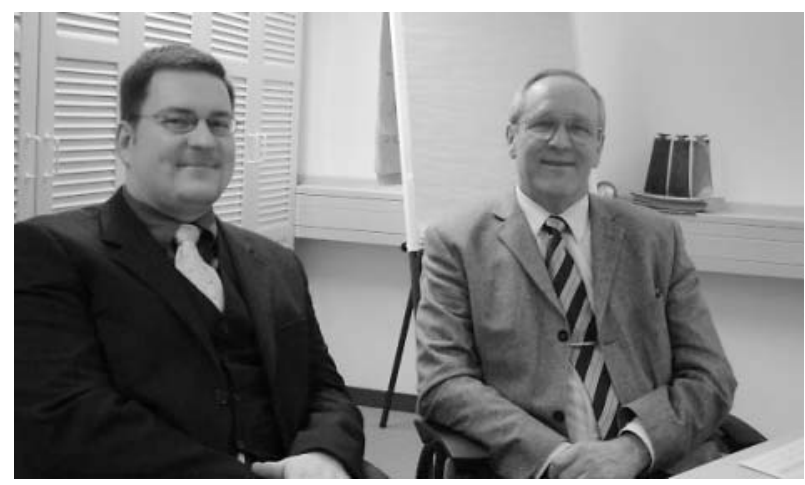

Abb. 2: Dipl.-Wirtschaftsing. (FH) Stephan Jahnke und Prof. Dipl.-Ing. Alfred Hentschel
Die HUCH GmbH, ein Familienunternehmen mit 75-jähriger Geschichte, stellt Druck- und drucklose Behälter aus Stahl in zylindrischer Form her, die sowohl flüssige als auch gasförmige Medien aufnehmen können. Die Behälter werden in mehreren Größen (von 75 bis 3000 l) und Ausfertigungen auf Basis der Kundenwünsche hergestellt. Die etwa 600 verschiedenen Erzeugnisse des Fertigungssortimentes können in 13 Erzeugnisgruppen eingeteilt werden. Die größte Bedeutung haben die Druckwasserkessel, Solarspeicher und Luftbehälter. Damit ist die HUCH GmbH vor allem Zulieferer für Pumpenhersteller und Anbieter von Solar- und Heiztechnik.

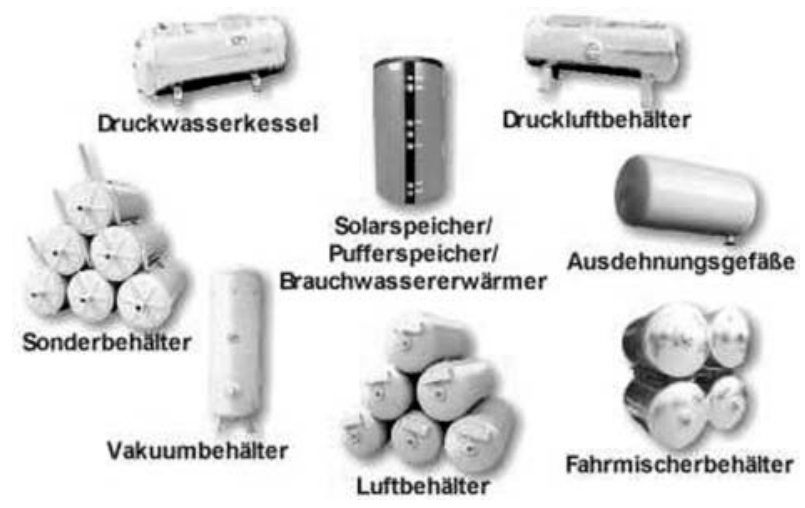

Abb. 3: Erzeugnisse der Fa. HUCH GmbH

Angesichts steigender Produktionszahlen und wirtschaftlicher Expansion in den letzten zehn Jahren sind auch die Anforderungen des Marktes in Bezug auf die Qualität der Produkte und die Flexibilität und Innovation der Fertigung gestiegen. Um den Erwartungen der Kunden hinsichtlich kurzer Lieferzeiten und Termintreue gerecht zu werden, ist es notwendig, den Produktionsablauf und im Speziellen die Produktionsvorbereitung systematisch zu planen und ständig zu optimieren. Der vermehrte Einsatz von Planungstechniken in Verbindung mit adäquater Rechentechnik wird somit auch in klein- und mittelständischen Unternehmen wie der HUCH GmbH mit ihren rund 50 Beschäftigten und einer Produktionsfläche von etwa $2.500 \mathrm{~m}^{2}$ unumgänglich.

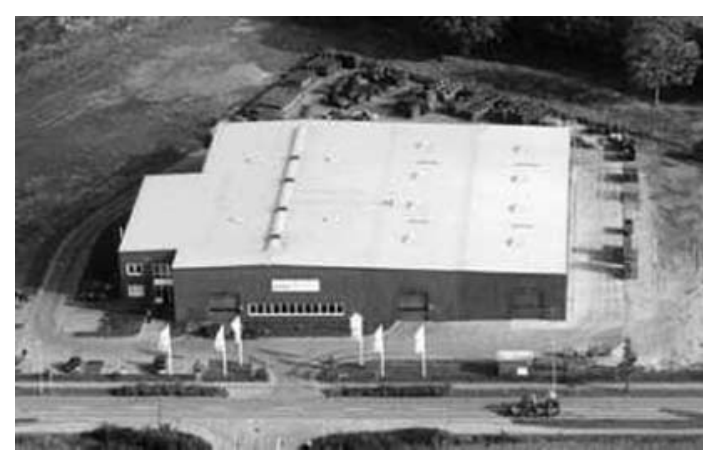

Abb. 4: Werk I der Fa. HUCH GmbH 
Die angefertigte Diplomarbeit steigt in diesen Veränderungsprozess in der Produktionsvorbereitung ein.

Es wurden grundlegende betriebliche Daten erstellt, die für die später beabsichtigte Implementierung in das vorhandene PPS-System unentbehrlich sind. Zunächst wurde der Ist-Zustand der betrachteten Produktionsstätte dargestellt und im Laufe dessen die vorhandenen Probleme und Schwachstellen aufgezeigt. Als Schwerpunkt in diesem Zusammenhang ist die Erfassung von arbeitsplatzbezogenen Kosten (Maschinenstundensätze) zu nennen.

Im Anschluss daran wurden aus dem Produktionsprogramm Typenvertreter ausgewählt, die das gesamte Produktspektrum repräsentieren. Für die Typenvertreter wurden die Arbeitsabläufe analysiert und Zeitmessungen durchgefuihrt. Da die aufgenommenen Zeiten stark von bestimmten Einflussgrößen abhingen, war die Anwendung von Planzeiten sinnvoll. Auf Basis des damit geschaffenen Zeitsystems in Form von Vorgabezeiten wurden dann die Arbeitspläne erstellt. Diese waren so gestaltet, dass sie als Typenarbeitsplan für jeden Behälter der jeweiligen Produktgruppe und für Neuentwicklungen als Arbeitsgrundlage genutzt werden können.

Die Arbeitspläne und vor allem die Festlegung von Zeiten (Vorgabezeitbestimmung) waren in diesem Zusammenhang der erste Schritt im Rahmen des Planungsprozesses in der Fertigung, ohne die eine weitergehende Planung von Auslastungen, Kapazitäten und Terminen gar nicht möglich war. Darüber hinaus ermöglichte die Erfassung der Zeiten je Einheit und der Rüstzeiten produktbezogene Kostenbetrachtungen. Dementsprechend mündeten die bisher gewonnenen Erkenntnisse in eine Kalkulation der Selbstkosten auf Basis von Maschinenstundensätzen, die zu einer Überprüfung des bestehenden Kalkulationsschemas fuihrten.

Die Grundlage für eine weiterfuihrende Betrachtung auf Teilkostenbasis war die Erfassung von fixen und variablen Stuickkosten. Im Ergebnis dessen wurden zunächst wirtschaftliche Losgrößen bestimmt, um die Fertigung von Aufträgen zu minimalen Herstellkosten zu realisieren. Durch eine anschließende Untersuchung der Dekkungsbeiträge und Gewinnschwellen für die ausgewählten Typenvertreter ließen sich dann objektive Aussagen über deren Wirtschaftlichkeit treffen.

Demzufolge werden hier Möglichkeiten aufgezeigt, wie neben den Marktforderungen an klein- und mittelständischen Unternehmen auch wirtschaftliche Aspekte bei der Zusammensetzung des Fertigungssortimentes stärkere Beachtung finden können.

Nun zu den einzelnen Ergebnissen der durchgefuihrten Untersuchungen: Durch die Anwendung des Kalkulationsschemas auf Basis von Maschinenstundensätzen wurde deutlich, dass einige Produktgruppen durch die pauschale Umlage der Fertigungskosten bisher zu hoch, andere wiederum zu niedrig kalkuliert wurden (Abweichung von +20 bis $-5 \%$ ). Dies ist mit der unterschied- lichen Inanspruchnahme der Arbeitsplätze zu erklären und zeigt deutlich, dass die Selbstkosten entsprechend dem tatsächlichen Werteverbrauch der Erzeugnisse kalkuliert werden müssen, um Unter- bzw. Überbewertungen zu vermeiden. Denn durch das bestehende Kalkulationsschema werden die Kosten der Maschine auf alle Erzeugnisse gleichmäßig in Form der Fertigungsgemeinkosten verteilt. Infolge der Erweiterung der differenzierenden Zuschlagskalkulation durch die Rechnung mit Maschinenstundensätzen erhöht sich dementsprechend die Objektivität der Selbstkosten. Damit kann die Wirtschaftlichkeit eines Erzeugnisses besser beurteilt werden. So ist es nun möglich, vor allem im Bereich der Vorkalkulation für neue Produkte relativ genau festzustellen, welche Kosten zu erwarten bzw. durch die Erlöse zu erwirtschaften sind.

Aus der Aufteilung der Selbstkosten in fixe und variable Bestandteile ist zu erkennen, dass den fixen Kosten bei der HUCH GmbH mit etwa 10 bis $20 \%$ nur sehr geringe Bedeutung zukommt. Die variablen Kosten dagegen bestimmen die Höhe der Selbstkosten entscheidend. Der größte Teil der variablen Kosten, die Materialkosten, nehmen somit in Bezug auf Kostensenkungspotenziale die höchste Priorität ein.

Die Ermittlung optimaler Losgrößen ergibt im Vergleich zur bisher gängigen Praxis in der HUCH GmbH, die Aufträge in Lose zu fünf oder zehn Einheiten aufzuteilen, starke Abweichungen. So schwanken die Stückzahlbereiche der optimalen Losgröße in den einzelnen Produktgruppen von 10 bis 60 Einheiten.

Die bedeutende Stellung der Materialkosten ist auch bei der Untersuchung der Deckungsbeiträge und Gewinnschwellen erkennbar. So weisen die Produkte der HUCH $\mathrm{GmbH}$ meist positive Deckungsbeiträge und eine Gewinnschwelle im positiven Stückzahlbereich auf. Die Gewinnzone wird also in vielen Fällen erreicht und eine wirtschaftliche Fertigung ist gegeben, mit Ausnahme der Produkte, welche einen extrem hohen Materialeinsatz bedingen. Hier muss es zu einer Reduzierung des Materialkostenanteils kommen, damit der Gewinn des Unternehmens nicht weiter belastet wird. Höhere Verkaufserlöse unterstuitzen zwar dieses Ziel, sind aber in einem stark kundenorientiertem Markt nicht in dem Maße durchsetzbar.

Aus den beschriebenen Betrachtungen ergibt sich ein Weg von aufeinander aufbauenden Arbeitsschritten, der eine durchgängige Planung in der Arbeitsvorbereitung und eine objektive Bewertung der Wirtschaftlichkeit der Fertigung ermöglicht. Die ganzheitliche Betrachtung des Planungsprozesses wird in Abbildung 5 noch einmal verdeutlicht.

Eine programmiertechnische Umsetzung dieser Problematik wurde der Arbeitsvorbereitung des Unternehmens in Form von zwei miteinander verknuipften Programmdateien auf Basis der Tabellenkalkulation „Excel“ zur Verfuigung gestellt. Damit entstand ein Hilfsmittel, durch dessen Anwendung für jedes Erzeugnis ein Arbeitsplan mit der Festlegung der Vorgabezeiten erstellt werden kann. Aufbauend darauf können die auf dieser Weise im 


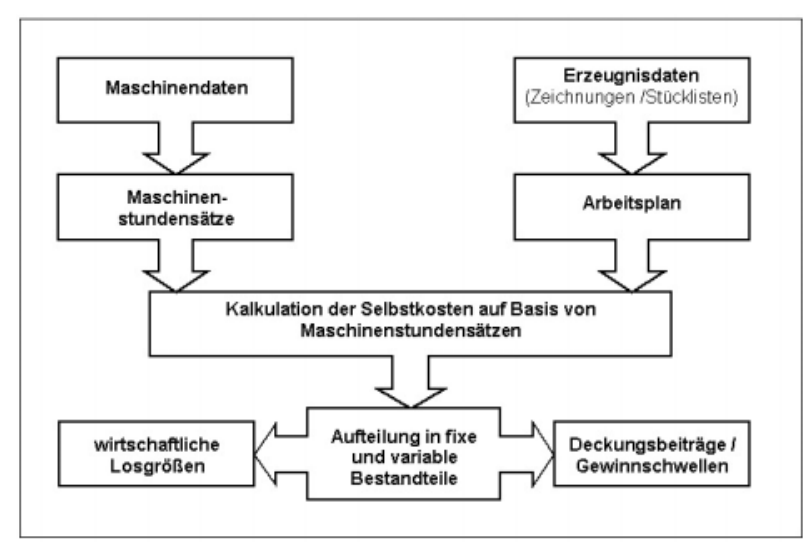

Abb. 5: Planungsprozess

Arbeitsplan zusammengefassten Daten und die auf Basis der Maschinendaten errechneten Maschinenstundensätze für eine Kalkulation der Selbstkosten mit Maschinenstundensätzen verwendet werden. Die dann wiederum die Grundlage für die weiteren Kostenbetrachtungen laut Abb. 5 bildet. Mit diesem Arbeitsmittel ist eine umfassende Betrachtung jedes Erzeugnisses in einer Datei möglich. Der Arbeitsaufwand wird so minimal. Die Excel-Datei, in der die Maschinenstundensätze berechnet werden, liefert die Datengrundlage für die Kalkulation der Selbstkosten. Die Maschinendaten sollten hier jährlich den betrieblichen Veränderungen angepasst werden. Die zweite Excel-Datei ist die Basis für die angestrebte Implementierung der Arbeitsplanung in das PPS-System und muss daher für jedes Erzeugnis erstellt werden. Danach ist zu überlegen, auf welche Art und Weise eine Zusammenfuihrung der einzelnen Erzeugnisdaten in eine Datenbank erfolgen muss.

Grundprinzip der Excel-Programmierung ist es, Daten, die einmal erfasst bzw. errechnet worden sind, jeweils für die folgenden Berechnungen weiter zu nutzen. Das bedeutet, dass die einmal erfassten Maschinendaten fuir die Berechnung der Maschinenstundensätze dienen. Diese werden dann im Rahmen der Kalkulation der Selbstkosten mit den jeweiligen Vorgabezeiten multipliziert. Bei der Aufteilung der Selbstkosten in fixe und variable Bestandteile werden dieselben Maschinenstundensätze wieder verwendet und getrennt jeweils mit Rüstzeit und Zeit je Einheit multipliziert. Aus diesem Berechnungsschema werden anschließend bei der Ermittlung der wirtschaftlichen Losgröße die soeben bestimmten fixen und variablen Herstellkosten verwendet. Letztlich finden die fixen und variablen Selbstkosten im Rahmen der Berechnung der Deckungsbeiträge und Gewinnschwellen Anwendung.

Damit wird deutlich, dass die Aufgabe für den Arbeitsplaner nur darin liegt, fuir jedes Erzeugnis die Produktdaten einzugeben und im Rahmen der Vorgabezeitbestimmung die arbeitsplatzspezifischen Einflussgrößen festzulegen. Die Ergebnisse (Arbeitsplan, Selbstkosten, Losgröße, Deckungsbeitrag und Gewinnschwelle) werden dann automatisch berechnet. Somit erscheinen mit der Anwendung dieses Schemas die Entscheidungen der Arbeitsvorbereitung nicht mehr isoliert, sondern fuigen sich vielmehr in ein Gesamtbild ein. Denk- und Hand- lungsweisen in der Arbeitsvorbereitung sollten damit tiefgreifend verändert werden. Die vielfältigen, auf den Fertigungsprozess einwirkenden Faktoren können nun im stärkeren Maße in die betrieblichen Entscheidungen mit einbezogen werden. Die zu treffenden Entscheidungen werden dadurch im stärkeren Maße an wirtschaftlichen Aspekten ausgerichtet und so besser nachvollziehbar. Dies stellt die Grundlage für den wirtschaftlichen Erfolg eines Unternehmens dar.

Im Folgenden soll noch einmal zusammenfassend erörtert werden, welche längerfristigen Maßnahmen sich für die HUCH GmbH aus der angefertigten Diplomarbeit ergeben und in der nächsten Zukunft sinnvollerweise umgesetzt werden sollten.

Die bisher gewonnenen Aussagen führen zu dem Schluss, dass sich die bestehende Situation in der HUCH $\mathrm{GmbH}$ durch die zukünftige Entwicklung stark verändern wird. So wird sich der allgemeine Arbeitsaufwand, der im Zusammenhang mit der Fertigung der Behälter, aber auch immer mehr mit der Verwaltung steht, auf Grund steigender Produktionszahlen erhöhen. Dieser Mehraufwand lässt sich mit einer Hebung der Arbeitsproduktivität (durch Optimierung in der Arbeitsorganisation und stärkere Automatisierung) und mit einer Aufstockung des Personalbestandes in ausgeglichenem Maße abdecken. Im Zuge dieser expansiven Entwicklung des Unternehmens verändert sich auch die Stellung im Markt. Das hat Auswirkungen auf die Beziehungen sowohl zu den wirtschaftlichen Partnern (Kunden, Lieferanten, Banken), als auch zu den Konkurrenten. Im Allgemeinen verbessert sich dann die Verhandlungsposition so, dass jetzt beispielsweise bei der Beschaffung auf Grund der gesteigerten Abnahmemenge günstigere Einstandspreise erreicht werden. Damit wachsen die wirtschaftlichen Möglichkeiten vor allem in Bezug auf die Preisgestaltung. Demzufolge wird der allmähliche Übergang von einem eher handwerklichen Klein-Unternehmen mit geringem wirtschaftlichem Einfluss hin zu einem mittleren Industrie-Unternehmen mit größerer Bedeutung vollzogen.

Um sich auf die angesprochenen Veränderungen einstellen zu können und damit den Erfolg des Unternehmens weiterzufuihren, sind geeignete Maßnahmen zu finden und umzusetzen. Zu diesem Zweck wurde ein Maßnahmenplan entwickelt, der die wichtigsten Erkenntnisse der Diplomarbeit zusammenfasst. Der nachfolgend abgebildete Maßnahmenplan beinhaltet Handlungsweisen, die vier Bereichen zugeordnet sind (s. Abb. 6). Die aufgefuihrten Maßnahmen sind Vorschläge, die eine innerbetriebliche Diskussion anregen sollen. Da Veränderungen im Unternehmen häufig mit einem Investitionsaufwand verbunden sind, ehe diese dann zu einer langfristigen Einsparung von Kosten führen, bleibt es natürlich der Geschäftsfuihrung des Unternehmens vorbehalten, welche Bestandteile letztlich verwirklicht werden.

Die Ziele der HUCH GmbH für die Zukunft sind klar gesteckt. Durch die Überschreitung der Schwelle, zu einem mittleren Industrieunternehmen heranzuwachsen, erreicht die steigende Komplexität der Fertigung einen 


\begin{tabular}{|c|c|c|}
\hline Bereich & Maßnahme & Wirkung \\
\hline $\begin{array}{l}\text { Organisation } \\
\text { / Personal }\end{array}$ & $\begin{array}{l}\text { - Verstärkung des Vertriebs } \\
\text { im Außendienst } \\
\text { - Verstärkung im } \\
\text { Lagerbereich } \\
\text { - Festlegung von Zuständig- } \\
\text { keiten und genaue } \\
\text { Verteilung der } \\
\text { Arbeitsaufgaben }\end{array}$ & $\begin{array}{l}\text { Entlastung der } \\
\text { Innendienstmitarbeiter } \\
\text { Entlastung der } \\
\text { Produktionsmitarbeiter } \\
\text { Verringerung von Fehlsteuerungen } \\
\text { und Mehrarbeit }\end{array}$ \\
\hline $\begin{array}{l}\text { Produktion / } \\
\text { Logistik }\end{array}$ & $\begin{array}{l}\text { - Verringerung des } \\
\text { manuellen Transportes } \\
\text { - einheitliche Transportmittel } \\
\text { - Einsatz von mehrstöckigen } \\
\text { Paletten-Regallagern, } \\
\text { anstatt der Bodenlagerung } \\
\text { im Freien } \\
\text { - einheitliche Lagerformen } \\
\text { - Erstellung von } \\
\text { Arbeitsplänen }\end{array}$ & $\begin{array}{l}\text { Verkürzung der Übergangszeiten } \\
\text { zwischen den Arbeitsplätzen } \\
\text { Abbau von Anpassungsproblemen } \\
\text { Erhöhung der Effektivität der } \\
\text { Lagerfläche } \\
\\
\text { Abbau von Anpassungsproblemen } \\
\text { verbesserte Planbarkeit der } \\
\text { Fertigung }\end{array}$ \\
\hline $\begin{array}{l}\text { Material- } \\
\text { wirtschaft }\end{array}$ & $\begin{array}{l}\text { - make-or-buy-Entscheidung } \\
\text { bei Materialbeschaffung } \\
\text { - Bestellmengen optimieren } \\
\text { - Losgrößen optimieren }\end{array}$ & $\begin{array}{l}\text { Senkung der Materialkosten } \\
\text { Senkung der Bestell-/Lagerkosten } \\
\text { Senkung der Herstellkosten }\end{array}$ \\
\hline $\begin{array}{l}\text { Kosten- } \\
\text { rechnung }\end{array}$ & $\begin{array}{l}\text { - Differenzierte } \\
\text { Kostengliederung nach } \\
\text { Kostenarten/-stellen } \\
\text { - Kalkulation der } \\
\text { Selbstkosten mit } \\
\text { Maschinenstundensätzen } \\
\text { - Untersuchung der } \\
\text { Deckungs-beiträge u. } \\
\text { Gewinnschwellen }\end{array}$ & $\begin{array}{l}\text { genauere Bestimmung der } \\
\text { Gemeinkostenzuschläge } \\
\text { Erhöhung der Objektivität } \\
\text { Überwachung der Wirtschaftlichkeit }\end{array}$ \\
\hline
\end{tabular}

Abb. 6: Maßnahmenplan

Grad, der eine manuelle Regelung der Fertigungsprozesse unmöglich macht. Daher müssen Planungstechniken zum Einsatz kommen, die den unweigerlich steigenden Arbeitsaufwand der unternehmerischen Tätigkeit durch Automatisierung abzudecken versuchen. Um den betrieblichen Werteverzehr besser überwachen und steuern und damit eine kostenminimale Fertigung realisieren zu können, muss ein effektives Kosten-Controlling eingefuihrt werden. Nur so kann die bisherige Erfolgsgeschichte der HUCH GmbH auch in der Zukunft fortgesetzt werden.

Um den allgemein steigenden Ansprüchen, denen sich die heutigen Unternehmen gegenübergestellt sehen, gerecht zu werden, ist es notwendig, ingenieurtechnische und betriebswirtschaftliche Sichtweisen zu kombinieren. So können Betriebswirte nur mit Kenntnis der technischen Hintergründe und Ingenieure nur auf der Grundlage von Kosteninformationen vernünftige Entscheidungen treffen. Mit der bereichsübergreifenden Betrachtung der Aufgaben in der Arbeitsvorbereitung versuchte die vorliegende Diplomarbeit dieser Richtung zu folgen und so anhand von Beispielen Hilfsmittel für die Lösung von Problemen in der HUCH GmbH zu entwickeln. So zeigten die Ergebnisse der Kostenbetrachtung, dass die bisher vom Unternehmen getroffenen Aussagen zu pauschal sind und zu falschen Entscheidungen führen können. Auch die Einsparung von Kosten kann nur dann zielgerichtet erfolgen, wenn der betriebliche Werteverzehr detailliert aufgeschlüsselt wird. Eine an die Diplomarbeit anknüpfende Auseinandersetzung mit den angearbeiteten Themen erfolgt in Form von weiteren Praktikanten- und Diplomandentätigkeiten in Zusammenarbeit mit der TFH Wildau.

\section{Autor}

Dipl.-Wirtschaftsing. (FH) Stephan Jahnke

Dieselstraße 54

14482 Potsdam

Sept. 1997 - 2001: Studium Wirtschaftsingenieurwesen an der TFH Wildau; Feb. - Juni 2001: Diplomarbeit bei der HUCH $\mathrm{GmbH}$; seit Juli 2001: Tätigkeit als Projektingenieur für Zeitwirtschaft/Logistik bei der ThyssenKrupp Umformtechnik GmbH Ludwigsfelde (Aufgabenbereich: Arbeitsstudium, Prozessrationalisierung; Durchführung von Ablauf-, Zeit- und Multimomentstudien; Neu- und Weiterentwicklung von Planzeitkatalogen; Erstellung von Kostenvergleichen/Wirtschaftlichkeitsrechnungen; Gestaltung und Optimierung der innerbetrieblichen Logistik; Angebotserstellung zu Transport und Verpackung)

\section{Betreuer der Diplomarbeit}

Prof. Dipl.-Ing. Alfred Hentschel

Technische Fachhochschule Wildau

Fachbereich Ingenieur-/Wirtschaftsingenieurwesen Labor Produktionstechnik/Produktionsvorbereitung Telefon + 493375 508-117, -133

E-Mail: hentsch@igw.tfh-wildau.de 